Jair Rodrigues Alves ${ }^{1}$

Heloísa Werneck Macedo 2

Alberto Novaes Ramos Jr. 3

Luiz Fernando Ferreira 4

Marcelo Luiz Carvalho Gonçalves 4

Adauto Araújo 4

\section{Parasitoses intestinais em região semi-árida do Nordeste do Brasil: resultados preliminares distintos das prevalências esperadas}

\author{
Intestinal parasite infections in a semiarid area \\ of Northeast Brazil: preliminary findings differ \\ from expected prevalence rates
}

1 Universidade do Estado do Piauí. Campus São Raimundo Nonato, Rua Iolanda Paixão 279, São Raimundo Nonato, PI 64770-000, Brasil. 2 Departamento de Patologia, Faculdade de Medicina, Universidade Federal Fluminense. Rua Marquês do Paraná 303, Niterói, RJ 24330-000, Brasil.

3 Departamento de Saúde Comunitária, Universidade Federal do Ceará. Rua Professor Costa Mendes 1608, Fortaleza, CE 60416-200, Brasil. 4 Departamento de Endemias Samuel Pessoa, Escola Nacional de Saúde Pública, Fundação Oswaldo Cruz. Rua Leopoldo Bulhões 1480, Rio de Janeiro, $R J$ 21041-210, Brasil. adauto@ensp.fiocruz.br

\begin{abstract}
We report on intestinal parasite infection prevalence in a population sample from São Raimundo Nonato, Southeast Piauí State, Brazil, aimed at comparison with previous studies on Trichuris trichiura and Ascaris lumbricoides infection. A total of 265 stool specimens were collected and examined by spontaneous sedimentation. Approximately 57\% of specimens were infected with at least one parasite species. Entamoeba coli (35.8\%), Endolimax nana (13.6\%), Hymenolepis nana (9.4\%), and hookworm (9.4\%) were the most frequently observed parasites. Two cases of roundworm infection were detected, probably acquired outside the region. T. trichiura eggs were not found. Interestingly, neither A. lumbricoides nor T. trichiura has been found in local prehistoric human coprolites. Nevertheless, hookworm infection has been present in the region for at least 7,000 years.
\end{abstract}

Key words Parasitic Intestinal Diseases; Helminthiasis; Coprological Survey; Parasitology

Resumo No presente trabalho procurou-se conhecer o perfil das enteroparasitoses na cidade de São Raimundo Nonato, sudeste do Piauí, e confirmar ou não os resultados obtidos em estudos anteriores em relação à infecção por Ascaris lumbricoides $e$ Trichuris trichiura. No período de setembro de 2000 a fevereiro de 2001, por meio de amostragem domiciliar por conveniência, foram examinadas 265 amostras fecais da população pelo método de sedimentação espontânea, das quais $57 \%$ foram positivas para enteroparasitos. Entamoeba coli (35,8\%), Endolimax nana (13,6\%), Hymenolepis nana $(9,4 \%)$ e os ancilostomídeos $(9,4 \%)$ foram os parasitos mais freqüentes. Foram observados dois casos de A. lumbricoides, possivelmente adquiridos fora do município. Nenhuma amostra foi positiva para T. trichiura. Esses resultados mostram um padrão diferente do restante do país. Traça-se um paralelo entre os resultados deste estudo com os achados paleoparasitológicos na população pré-histórica, habitante da região há pelo menos sete mil anos.

Palavras-chave Enteropatias Parasitárias; Helmintíase; Inquérito Coprológico; Parasitologia 


\section{Introdução}

No sudeste piauiense, pouco se conhece sobre a prevalência das parasitoses intestinais. Em estudos coprológicos preliminares, realizados em pequenos povoados do interior do Parque Nacional Serra da Capivara nos anos de 1984 e 1986, notou-se a ausência de Ascaris lumbricoides e de Trichuris trichiura em 485 pessoas examinadas (dados não publicados). Mais recentemente, em município limítrofe ao norte do Parque, não foram encontrados ovos de $A$. lumbricoides, apesar da ocorrência de protozoários em 2 a $25 \%$ e de helmintos em 0 a $2 \%$ das amostras examinadas. Foi feito o diagnóstico de tricuríase em apenas duas crianças, ambas recém chegadas de outros Estados (Ramos Jr. et al., 2000).

A ascaridíase, a ancilostomíase e a tricuríase representam as parasitoses intestinais mais freqüentes no país (Rey, 2001). Na década de 70, o inquérito realizado pela Superintendência de Campanhas de Saúde Pública/Ministério da Saúde (SUCAM) em 21 Estados, mostrou que A. lumbricoides e T. trichiura apresentaram as maiores freqüências relativas, $52,6 \%$ e 36,6\%, respectivamente (SUCAM, 1973). Em 1950, um estudo mostrou que o Piauí apresentava, dentre os Estados estudados, o maior índice de infecção por ancilostomídeos $(68,8 \%)$ e terceiro maior por A. lumbricoides (88,0\%) (Pellon \& Teixeira, 1950). Em recente estudo no Município de Parnaíba, região litorânea do Piauí, $A$. lumbricoides $(64,9 \%)$, ancilostomídeos $(16,5 \%)$, Entamoeba coli (43,3\%) e Entamoeba histolyticaldispar $(23,4 \%)$ foram os parasitos mais freqüentes (Oliveira et al., 2001).

No presente trabalho procurou-se avaliar de forma preliminar a freqüência de enteroparasitoses em localidades da área urbana da cidade de São Raimundo Nonato, localizada no entorno do Parque Nacional Serra da Capivara, sudeste do Piauí, e compará-la com os resultados obtidos anteriormente relacionados à infecção por A. lumbricoides e T. trichiura, que nesta região apresentaram ocorrência diferente das demais regiões do país (Ramos Jr. et al., 2000).

\section{Materiais e métodos}

O estudo foi realizado no período de setembro de 2000 a fevereiro de 2001, no Município de São Raimundo Nonato, localizado a $520 \mathrm{~km}$ de Teresina, região semi-árida do Estado, onde predomina a vegetação de caatinga. A população do município é de 26.880 habitantes, dos quais $17.201(63,4 \%)$ residem na zona urbana (IBGE, 2000).

Foram selecionados, nessa fase preliminar, quatro bairros periféricos da cidade: Paraíso das Aves, Bairro Vermelho, Santa Luzia e Baixão da Guiomar. Esses bairros são geograficamente contíguos, com padrões sócio-econômicos e educacionais semelhantes. A escolha desses bairros foi definida tomando-se como critério áreas onde foram iniciadas as atividades de visitação pelo Programa Municipal de Agentes Comunitários de Saúde (PACS) e do Programa Saúde da Família (PSF).

Após o consentimento informado pelo responsável de cada família, procedeu-se a aplicação do questionário individual no próprio domicílio, realizada por um dos autores, por meio do qual obtinham-se informações sobre o sexo, a idade, o local da residência e a ocorrência de viagens para fora do município.

Nos bairros selecionados, foi obtida por conveniência uma amostra de famílias para a avaliação parasitológica. Em cada família selecionada, os indivíduos que consentiram participar do estudo receberam um frasco plástico para coleta das fezes, contendo substância conservante (MIF: mercúrio + iodo + formol), com a identificação de cada participante. As amostras foram recolhidas dois a quatro dias após a distribuição dos frascos e permaneceram no MIF até serem examinadas. Foram coletadas 265 amostras fecais, uma de cada indivíduo, correspondendo a 1,54\% da população urbana do município. A média de idade dos indivíduos que participaram deste estudo foi de 23 anos, variando de 1 a 89 anos, sendo 60,5\% (155) do sexo feminino.

Os exames parasitológicos das amostras foram realizados no laboratório do Hospital Senador Cândido Ferraz, localizado no próprio município, e no Laboratório de Parasitologia do Hospital Antonio Pedro, da Universidade Federal Fluminense, na cidade de Niterói, Rio de Janeiro. Por dificuldades operacionais foi utilizado apenas o método de sedimentação espontânea de Lutz (1919), com leitura de três lâminas de cada amostra.

Este trabalho esteve de acordo com os princípios éticos contidos na Declaração de Helsinki e foi aprovado pelo Comitê de Ética da Escola Nacional de Saúde Pública/Fundação Oswaldo Cruz.

\section{Resultados}

Foram encontrados enteroparasitos em 57\% das amostras. E. coli $(35,8 \%)$, E. nana $(13,6 \%)$, 
H. nana $(9,4 \%)$ e os ancilostomídeos $(9,4 \%)$ foram os parasitos mais freqüentes. Apenas duas amostras foram positivas para A. lumbricoides e nenhuma para T. trichiura (Tabela 1).

\section{Discussão}

Todos os indivíduos que participaram deste trabalho receberam o resultado do exame parasitológico. Aqueles com diagnóstico de parasitose intestinal foram encaminhados às unidades de saúde do município para tratamento específico. Os resultados mostram infecções por protozoários e helmintos na região com ocorrência semelhante às encontradas em João Costa, município vizinho (Ramos Jr. et al., 2000).

Chama a atenção a ausência da infecção por T. trichiura e a baixa freqüência de A. lumbricoides. Os dois casos de ascaridíase foram adquiridos, provavelmente, fora do município. Uma das pessoas informou que havia recentemente chegado da cidade de São Paulo, onde passou pouco mais de um ano, e a outra que fazia viagens constantes a Petrolina, Pernambuco. Os familiares de ambos foram negativos para este parasito. Importante ressaltar que não há referência a tratamentos em massa da população com anti-helmínticos. Por contraste, essas parasitoses foram encontradas no $\mathrm{Mu}$ nicípio de Parnaíba, no litoral do Estado, onde $64,9 \%$ das amostras foram positivas para $A$. lumbricoides e $4,7 \%$ para $T$. trichiura (Oliveira et al., 2001).

A disseminação das helmintíases na região nordeste do país está em estreita dependência com a umidade do solo. Considera-se que nas regiões semi-áridas a longa estação seca é uma das circunstâncias limitantes para a proliferação de parasitos (Pessoa, 1959; Rey, 2001). Embora se possa argumentar que o ambiente seco dificulte a manutenção da infecção por A. lumbricoides e T. trichiura na população, o encontro relativamente freqüente de ancilostomídeos nas amostras indica um ambiente não de todo inóspito aos geohelmintos, existindo ainda condições propícias para a manutenção da infecção por protozoários intestinais.

Outro aspecto a ser ressaltado é a infecção por uma espécie ainda não descrita de Trichuris em roedores endêmicos do semi-árido $(\mathrm{Ke}$ rodon rupestris), assinalada há $32 \mathrm{mil}$ anos em fezes fossilizadas deste animal, persistente até

\begin{tabular}{|c|c|c|}
\hline \multicolumn{3}{|c|}{$\begin{array}{l}\text { Freqüência e percentual de parasitos intestinais. } \\
\text { São Raimundo Nonato, Piauí, Brasil, setembro } \\
\text { de } 2000 \text { a fevereiro de } 2001 \text {. }\end{array}$} \\
\hline Parasito & Freqüência & $\%$ \\
\hline Entamoeba coli & 95 & 35,8 \\
\hline Endolimax nana & 36 & 13,6 \\
\hline Hymenolepis nana & 25 & 9,4 \\
\hline Ancilostomídeos & 25 & 9,4 \\
\hline Giardia duodenalis & 23 & 8,7 \\
\hline $\begin{array}{l}\text { Entamoeba histolytical } \\
\text { Entamoeba dispar }\end{array}$ & 18 & 6,8 \\
\hline Blastocystis hominis & 9 & 3,4 \\
\hline Enterobius vermicularis & 4 & 1,5 \\
\hline lodamoeba butschlii & 2 & 0,8 \\
\hline Ascaris lumbricoides & 2 & 0,8 \\
\hline Taenia sp. & 1 & 0,4 \\
\hline
\end{tabular}

8 mil anos e desaparecendo a partir de então. Esse desaparecimento coincide com mudanças climáticas importantes que tornaram a região mais seca. Embora o hospedeiro ainda seja abundante na região do parque, o parasito não se manteve (Ferreira et al., 1991). Especula-se se o mesmo possa ter ocorrido com T. trichiura e o hospedeiro humano.

Em observações preliminares na população residente em vários povoados do entorno do Parque Nacional Serra da Capivara (Zabelê, Sítio do Mocó, Vargem Grande e outros), feitas desde 1984 e não publicadas, observou-se igualmente a ausência de $A$. lumbricoides e T. trichiura. Pretende-se seguir com inquéritos parasitológicos, ampliando-se concentricamente a área estudada até que ocorra a mudança do padrão, buscando-se explicar a ausência, ou a raridade na região, de duas helmintíases que apresentam as mais altas prevalências no país.

Os dados na população humana pré-histórica e na atual da região do Parque Nacional Serra da Capivara mostram, até o momento, padrões diferentes dos encontrados em outras regiões próximas. Chama particularmente a atenção a permanência da infecção por ancilostomídeos há pelo menos 7 mil anos (Araújo \& Ferreira, 1997), num ambiente desprovido de infecção por A. lumbricoides e T. trichiura. 


\section{Agradecimentos}

Financiamento: Programa Institucional de Bolsas de Iniciação Científica, Conselho Nacional de Desenvolvimento Científico e Tecnológico (CNPq)/Fundação Oswaldo Cruz (FIOCRUZ); Programa de Apoio a Núcleos de Excelência, CNPq; Programa de Apoio à Pesquisa Estratégica em Saúde (PAPES), FIOCRUZ; Escola de Governo, Escola Nacional de Saúde Pública, FIOCRUZ.

\section{Referências}

ARAÚJO, A. \& FERREIRA, L. F., 1997. Homens e parasitos: A contribuição da paleoparasitologia para a questão da origem do homem na América. Revista da Universidade de São Paulo, 34:58-70.

FERREIRA, L. F.; ARAÚJO, A.; CONFALONIERI, U.; CHAME, M. \& GOMES, D. C., 1991. Trichuris eggs in animal coprolites dated from 30,000 years ago. Journal of Parasitology, 77:491-493.

IBGE (Fundação Instituto Brasileiro de Geografia e Estatística), Censo Demográfico de 2000. <http:// www.ibge.gov.br/censo/>.

LUTZ, A., 1919. O Schistosomum mansoni e a schistosomatose segundo observações feitas no Brasil. Memórias do Instituto Oswaldo Cruz, 11:121-155.

OLIVEIRA, F. M.; COSTA, S. T. C. \& BEZERRA, F. S. M., 2001. Incidência de enteroparasitoses na zona rural do Município de Parnaíba, Piauí. Revista Brasileira de Análises Clínicas, 33:45-48.

PELLON, A. B. \& TEIXEIRA, I., 1950. Distribuição Geográfica da Esquitossomose Mansônica no Brasil. Rio de Janeiro: Divisão de Organização Sanitária.

PESSOA, S. B., 1959. Considerações sobre as verminoses no nordeste brasileiro. Revista do Instituto de Medicina Tropical de São Paulo, 1:57-80.

RAMOS Jr., A. N.; MACEDO, H. W.; CHIEFFI, P. P.; GONÇALVES, M. L. C.; CARVALHO, D. M.; ALVES J. R. ; FERREIRA, L. F. \& ARAÚJO, A., 2000. Sobre o resultado de exames parasitológicos de fezes em povoados do entorno do Parque Nacional Serra da Capivara, sudeste do Piauí: Um paradoxo. Saúde Coletiva, 5:468-469.

REY, L., 2001. Parasitologia. Rio de Janeiro: Editora Guanabara Koogan

SUCAM (Superintendência de Campanhas de Saúde Pública), 1973. Campanhas Contra Ancilostomose e Esquistossomose. Brasilia: SUCAM.

Recebido em 17 de abril de 2002

Versão final reapresentada em 15 de julho de 2002 Aprovado em 5 de setembro de 2002 